\title{
An Analysis of Nanoparticle Settling Times in Liquids
}

\author{
D. D. Liyanage, ${ }^{1}$ Rajika J. K. A. Thamali, ${ }^{1}$ A. A. K. Kumbalatara, ${ }^{1}$ \\ J. A. Weliwita, ${ }^{2}$ and S. Witharana ${ }^{1,3}$ \\ ${ }^{1}$ Department of Mechanical and Manufacturing Engineering, University of Ruhuna, Galle, Sri Lanka \\ ${ }^{2}$ Department of Mathematics, University of Peradeniya, Kandy, Sri Lanka \\ ${ }^{3}$ Sri Lanka Institute of Nanotechnology, Colombo, Sri Lanka
}

Correspondence should be addressed to S. Witharana; switharana@ieee.org

Received 10 September 2015; Revised 28 December 2015; Accepted 6 January 2016

Academic Editor: P. Davide Cozzoli

Copyright (C) 2016 D. D. Liyanage et al. This is an open access article distributed under the Creative Commons Attribution License, which permits unrestricted use, distribution, and reproduction in any medium, provided the original work is properly cited.

\begin{abstract}
Aggregation and settling are crucial phenomena involving particle suspensions. For suspensions with larger than millimeter-size particles, there are fairly accurate tools to predict settling rates. However for smaller particles, in particular micro-to-nanosizes, there is a gap in knowledge. This paper develops an analytical method to predict the settling rates of micro-to-nanosized particle suspensions. The method is a combination of classical equations and graphical methods. Validated using the experimental data in literature, it was found that the new method shows an order of magnitude accuracy. A remarkable feature of this method is its ability to accommodate aggregates of nonspherical shapes and of different fractal dimensions.
\end{abstract}

\section{Introduction}

Nanoparticles have drawn interest from scientific communities across a broad spectrum for their unusual magnetic, optical, thermal, and transport properties. Among these is the thermal conductivity that has been extensively studied and debated over the past two decades. For instance, the addition of traces of nanoparticles, often less than $1 \mathrm{vol} \%$, to a common heat transfer liquid like water, has demonstrated an increase in thermal conductivity by up to $40 \%$ [1-3]. Despite adding to excitement, such high degrees of enhancement surpass the predictions by classical theories. However there are a few contradictory observations too. In the famous INPBE experiment [4], the measured thermal conductivity enhancements were observed to be within the range predicted by the effective medium theories. These nanoparticle-liquid heat transfer blends are popularly known as nanofluids.

Particles suspended in liquids are prone to form aggregates that would finally lead to separation and settling due to gravity. On the other hand, aggregation is contemplated as a major mechanism responsible for the enhanced thermal conductivity demonstrated by nanofluids [5]. A certain degree of aggregation in a nanofluid may therefore be beneficial but the ultimate settling would limit its practical use. In addition to the deterioration of thermal conductivity, separated large aggregates may clog filters and block the flow in narrow channels in heat transfer devices. However, for mineral extraction and effluent treatment industries, separation and settling are basic prerequisites of operation. To welcome or to avoid it, one may need to understand the particle settling processes and settling rates. Having said so, only a few experimental studies have been hitherto dedicated to investigate the aggregation and settling dynamics of nanoparticles [6-8]. Even for microsized particles, only a few methods are available in literature to calculate the settling velocities [9]. Complex nature of aggregating nanoparticulate systems and difficulties in taking accurate measurements seem to be major challenges for the progress of experimental work $[6,10,11]$.

This paper puts forward analytical method to calculate the nanoparticle aggregation and settling times in liquids. To start the computation sequence, one should know the particle concentration in the nanofluid. In a situation of unknown particle concentration, one could measure the viscosity of the nanofluid and compute it as suggested by Chen et al. [12,13]. First step is to determine the aggregation time. For this a correlation is derived by taking into account all governing parameters. Second step is to determine the settling time. For this a new method is proposed, which is a combination of 
known equations, graphs, and estimations. The total time for settling should be the sum of the aggregating time and the settling time determined this way.

Lastly the proposed analytical method is validated with the experimental data for nanoparticle settling available in literature.

\section{Materials and Methods}

In this section, computational sequences are introduced to estimate the nanoparticle aggregation time and the aggregate settling time.

2.1. Method to Estimate the Nanoparticle Aggregation Time. Consider a colloidal system where nanoparticles are well dispersed in a liquid. Gradually the nanoparticles will start to form aggregates driven by a number of parameters such as nanoparticles size and concentration, solution temperature, stability ratio, and the fractal dimensions of objects [15-18]. At any given time $t$, these aggregates can be characterized by radius of gyration $\left(R_{a}\right)$ as follows:

$$
\frac{R_{a}}{r_{p}}=\left(1+\frac{t}{t_{p}}\right)^{1 / d_{f}}
$$

where $d_{f}$ is the fractal dimension of the nanoparticle aggregates, which is practically found to be in the range of 2.5-1.75 $[10]$. And the aggregation time constant $t_{p}$ is defined as [11]

$$
t_{p}=\frac{\left(\pi \mu r_{p}^{3} W\right)}{\left(k_{B} T \emptyset_{p}\right)} .
$$

Here $k_{B}, T, \emptyset_{p}$, and $\mu$ are, respectively, the Boltzmann constant, temperature, nanoparticle volume fraction, and viscosity of the liquid.

The stability ratio $W$ is defined by

$$
W=2 r_{p} \int_{0}^{\infty} \frac{B(h) \exp \left\{\left(V_{R}+V_{A}\right) / k_{B} T\right\}}{(h+2 r)^{2}} d h,
$$

where $B(h)$ is the parameter that captures the hydrodynamic interaction.

After Chen et al. [12], for interparticle distance $h$,

$$
B(h)=\frac{6\left(h / r_{p}\right)^{2}+13(h / a)+2}{6\left(h / r_{p}\right)^{2}+4(h / a)} .
$$

Moreover, $V_{R}$ is the repulsive potential energy given by

$$
V_{R}=2 \pi \epsilon_{r} \epsilon_{0} r_{p} \Psi^{2} \exp (-\Lambda h)
$$

for dielectric constant of free space, $\epsilon_{0}$, and $\zeta$ potential, $\Psi$. Note that this expression is valid when $\Lambda r_{p}<5$.

Debye parameter is given by $\Lambda=5.023 \times 10^{11} I^{0.5} /\left(\epsilon_{r} T\right)^{0.5}$, where $\epsilon_{r}$ is the relative dielectric constant of the liquid and $I$ is the concentration of ions in water. In the absence of salts, $I$ and $\mathrm{pH}$ relate in the form of $I=10^{-\mathrm{pH}}$ for $\mathrm{pH} \leq 7$ and $I=$ $10^{-(14-\mathrm{pH})}$ for $\mathrm{pH}>7$.
Also the attractive potential energy is defined as

$$
\begin{aligned}
V_{A} & =-\frac{A}{6}\left[\frac{2 r_{p}{ }^{2}}{h\left(h+4 r_{p}\right)}+\frac{2 r_{p}{ }^{2}}{\left(h+2 r_{p}\right)^{2}}\right. \\
& \left.+\ln \left(\frac{h\left(h+4 r_{p}\right)}{\left(h+2 r_{p}\right)^{2}}\right)\right],
\end{aligned}
$$

where $A$ is the Hamaker constant.

Using above set of equations and calculation procedure, one could compute the time taken to form an aggregate $\left(t_{p}\right)$ of a known radius $\left(R_{a}\right)$.

2.2. Method to Estimate the Aggregate Settling Time. Terminal settling velocity of a particle in a fluid body is governed by multiple factors such as fluid density and particle density and size and shape and concentration and degree of turbulence and solution temperature [19]. The Stokes law of settling was originally defined for small, $\mathrm{mm}$, or $\mu \mathrm{m}$ size spherical particles with low Reynolds Numbers $(\operatorname{Re} \leq 0.3)$. The drag force of a creeping flow over a rigid sphere consists of two components, namely, pressure drag $\left(F_{p}=\pi \mu d U\right)$ and the shear stress $\operatorname{drag}(F=2 \pi \mu d U)[18,20,21]$. Thus the total drag becomes $F_{D}=3 \pi \mu d U$. Using the Stokes equation, a spherical particle moving under ideal conditions of infinite fluid volume, lamina flow, and zero acceleration can be expressed in the form

$$
\frac{4 \pi R^{3}\left(\rho_{p}-\rho_{f}\right) g}{3}-6 \pi \mu U R=0,
$$

where $U$ is terminal velocity and $R$ is the equivalent radius of the aggregate. Stokes drag $(6 \pi \mu U R)$ could be reexpressed as $F_{\mathrm{SD}}=C_{D}\left(\rho_{f} U^{2} / 2\right) A$, where $C_{D}=F^{\prime} /\left(\rho_{f} U^{2} / 2\right)$ for force $F^{\prime}$ per unit projected area and $A$ is the projected area of the particle to incoming flow. For a sphere, $A=4 \pi R^{3} / 3$. Value for $C_{D}$ can be found from Figure 3 . Note that a particle in a creeping flow where Reynolds Number is very small tends to face the least projected area to the flow [14].

Thus (X) becomes $4 \pi R^{3}\left(\rho_{p}-\rho_{f}\right) g / 3-C_{D}\left(\rho_{f} U^{2} / 2\right) A=0$ and, hence,

$$
U=\sqrt{\frac{8 \pi R_{a}^{3}\left(\rho_{p}-\rho_{f}\right) g}{3 C_{D} \rho_{f} A}} .
$$

However, a nanoparticle will hardly qualify for the Stokes conditions because of its larger surface area-to-volume ratio. In these circumstances, the surface forces dominate over gravitational forces. Also, for a nanoparticle dispersed in a liquid, the intermolecular forces (Van der Waal's, iron-iron interactions, iron-dipole interactions, dipole-dipole interactions, induced dipoles, dispersion forces, and overlap repulsion) along with the thermal vibrations (Brownian motion) and diffusivity will take over the Newtonian forces [22, 23]. Hence the gravitational force does not dominate the settling velocity anymore. Thus the nanoparticles in suspension will 
have a random motion, not only vertically downward. Recall that we assumed that the nanoparticles do not start noticeable settling till they made aggregates of a sufficient mass. Experimental data shows that the nanosize particles $(1 \mathrm{~nm}-20 \mathrm{~nm})$ form microsize aggregates $(0.1-15 \mu \mathrm{m})[10]$. The shapes of the aggregates depend upon fractal dimension $\left(d_{f}\right)$ that typically varies between 1.5 and 2.5 in most cases. As $d_{f}$ gets closer to 3 , the shape of aggregates approaches a spherical shape. Also, when a colony of nanoparticles form one microsize aggregate, the size factor comes into effect, the intermolecular forces disappear, and the Newtonian forces begin to dominate on the aggregate [24]. For instance, mean free path $(L=\sqrt{2 k T / 3 \pi x \mu t})$ due to Brownian motion shortens by $86.44 \%$ when nanoparticles of $23 \mathrm{~nm}$ come together and form $2.5 \mu \mathrm{m}$ aggregate [25]. Following assumptions are made for application of Stokes law for the present work.

(a) Reynolds Number (Re). Aggregates have very low settling velocities, and thus they give very small Reynolds Numbers.
For example, Re can be expected in the region of $0.001 \sim 0.0001$ [26]:

$$
\operatorname{Re}=\frac{D_{a U} \rho_{f}}{\mu} .
$$

The diameters of settling aggregates were determined in Section 2.1 above. With this information alone the derivation of Re of these objects is still not possible. Their settling velocities too are required but not known. Therefore an iterative method is proposed to estimate Re [27]:

$$
C_{D} \operatorname{Re}^{2}=\frac{4 g\left(\rho_{p}-\rho_{f}\right) \rho_{f} d^{3}}{3 \mu^{2}}
$$

Figure 1 was constructed for (8) and (9). This enables determination of Re using the aggregate diameter.

(b) Sphericity $(\Psi)$. Sphericity $(\Psi)$ indicates how spherical the aggregate is. This is defined by

$$
\text { Sphericity }(\Psi)=\frac{\text { surfce area of a sphere }}{\text { surface area of the aggregate which has the same volume of the sphere }} \text {. }
$$

Rhodes [20] developed graphs to correlate $\mathrm{Re}$ and $C_{D}$ for different values of sphericity $(\Psi)$. These graphs are shown in Figure 2. However the aggregates of smaller sizes have very low Reynolds Numbers in the order of $10^{-4} \sim 10^{-6}$ as stated before. In order to capture values of $C_{D}$ for such low Reynolds Numbers, graphs were extended to the left-hand side. The extended graphs are presented in Figure 3. Equations corresponding to the set of graphs are listed in Table 1.

To use Figure 3, one needs to know the sphericity $(\Psi)$ of aggregates. This information is given in Table 2 for common and general shapes $[17,28,29]$.

(c) Density of the Aggregate. Smoluchowski model states that nanoparticles clustered together form a complete sphere with voids inside [11, 18]. Moreover, when fractural dimension decreases, the aggregate geometry gets closer to a twodimensional flat object. In this case $d_{f}$ would approach a value of 1.8 and appears like the shape in Figure 4(a).

Now consider the density of a settling aggregate. This flat object, shown in Figure 4(a), is surrounded by a thin layer of liquid molecules. Density of the settling aggregate can thus be safely assumed as equal to the density of the solid.

(d) Density of Suspension. When preparing a nanofluid, the suspension is thoroughly stirred for the particles to be distributed evenly in the container. Similarly it is assumed that the aggregates too are evenly distributed throughout the liquid. Thus this system portrays a homogenous flow of aggregates. The density of this solid-liquid mixture is determined using

$$
\rho_{m}=\emptyset_{a} \rho_{a}+\left(1-\emptyset_{a}\right) \rho_{f}
$$

where $\emptyset_{a}$ is the aggregate volume fraction given by

$$
\emptyset_{a}=\emptyset_{p}\left(\frac{R_{a}}{r}\right)^{3-d_{f}} .
$$

(e) Viscosity of the Suspension. Maxwell Garnett [25] put forward the following relationship to calculate the viscosity of suspensions where the particle volume concentration is less than 5\%:

$$
\mu=\mu_{0}\left(1+2.5 \emptyset_{p}\right)
$$

(f) Zero-Slip Condition and Smooth Surface. When nanoparticles are dispersed in water, the water molecules make an orderly layer around the nanoparticle, a phenomena known as liquid layering $[12,30]$. The water layer directly in contact with the nanoparticle gets denser than the bulk liquid further away. Due to this particle-water bond at the boundary, it is reasonable to assume a no-slip region for water. Further, the surface of the aggregate is smooth and therefore the drag due to roughness of the aggregate may not come into effect [31]:

$$
F_{A}=6 \pi \mu_{b} R_{a} U_{a}\left(\frac{2 \mu_{b}+R_{a} \beta_{a b}}{3 \mu_{b}+R_{a} \beta_{a b}}\right),
$$

where $\mu_{b}$ and $\beta_{a b}$ are viscosity of the liquid and the coefficient of sliding friction. When there is no tendency for slipping $\beta_{a b} \approx \infty$ and therefore the above expression retracts to the Stokes law, $F_{A}=6 \pi \mu_{b} R_{a} U_{a}$. Hence $(\mathrm{X})$ can be used.

(g) Batch Settling. Originally the Stokes law is for a sphere travelling in infinite medium at low Re. However, in the aggregation and settling systems studied in this work, 
TABLE 1: Equations of logarithm of drag coefficients $\left(C_{D}\right)$ with respect to logarithm of Re for different values of sphericity $(\Psi)$ extracted from graphs in Figure 2.

\begin{tabular}{lc}
\hline$\Psi$ & Relationship of drag coefficient $\left(C_{D}\right)$ and low Reynolds Numbers $(\mathrm{Re})$ \\
\hline 0.125 & $\log \left(C_{D}\right)=0.1202 \log (\mathrm{Re})^{2}-0.6006 \log (\mathrm{Re})+2.032$ \\
0.22 & $\log \left(C_{D}\right)=0.0043161 \log (\mathrm{Re})^{3}+0.9725 \log (\mathrm{Re})^{2}-0.68 \log (\mathrm{Re})+1.937$ \\
0.6 & $\log \left(C_{D}\right)=0.0067 \log (\mathrm{Re})^{3}+0.083 \log (\mathrm{Re})^{2}-0.73 \log (\mathrm{Re})+1.8$ \\
0.806 & $\log \left(C_{D}\right)=0.0099 \log (\mathrm{Re})^{3}+0.0697 \log (\mathrm{Re})^{2}-0.7906 \log (\mathrm{Re})+1.7225$ \\
1 & $\log \left(C_{D}\right)=0.0116 \log (\mathrm{Re})^{3}+0.05793 \log (\mathrm{Re})^{2}-0.8866 \log (\mathrm{Re})+1.443$ \\
\hline
\end{tabular}

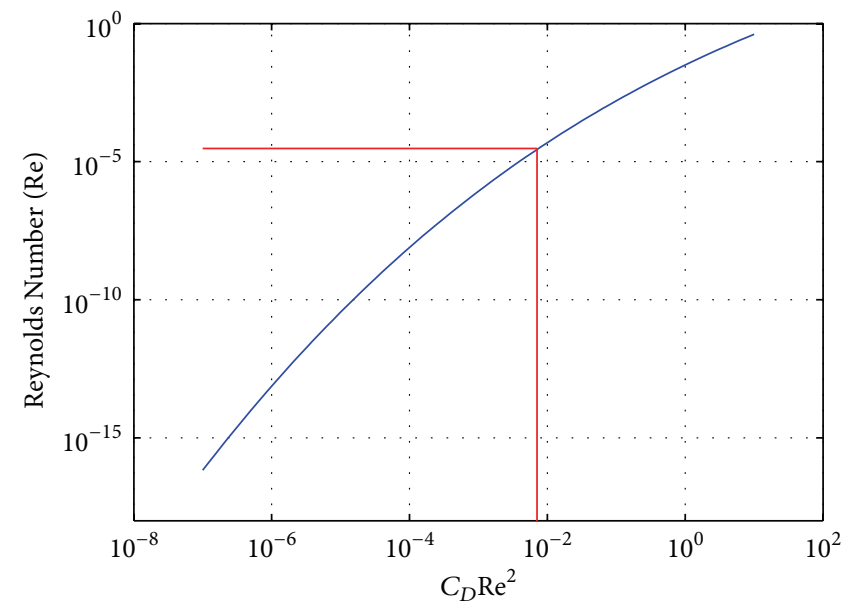

Figure 1: Re versus $C_{D} \operatorname{Re}^{2}$ for spherical particles.

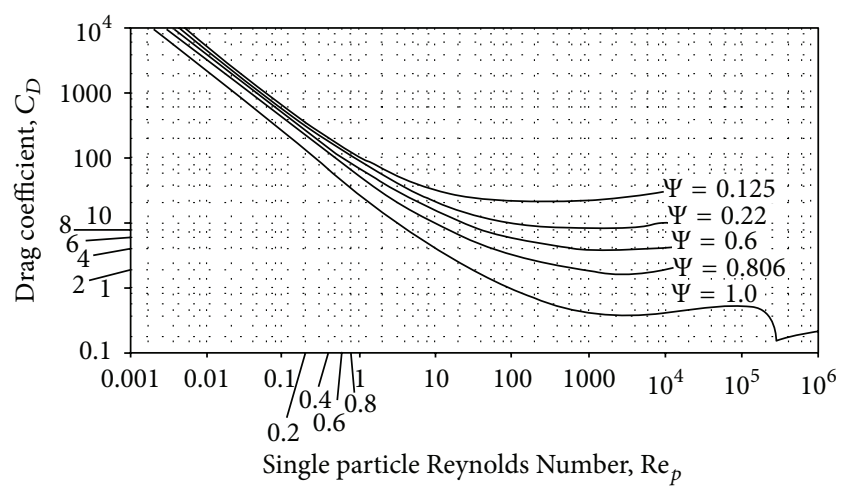

Figure 2: Drag coefficient $\left(C_{D}\right)$ and particle Reynolds Number $\left(\operatorname{Re}_{p}\right)$ for different sphericity $(\Psi)[14]$.

the particles are in large number in a finite volume of liquid. Those close proximity aggregates are obviously influenced by each other and may deviate from the Stokes law. Richardson and Zaki [32] defined the term batch settling velocity $\left(U_{p}\right)$ or particle superficial velocity for an event where there are a number of particles in a finite volume. When $\operatorname{Re}<0.3, U_{p}=$ $U_{T} \varepsilon^{\prime 4.65}$, where $\varepsilon^{\prime}$ is liquid void fraction expressed as $\varepsilon^{\prime}=$ Voids Volume/Total Volume. Here $\varepsilon^{\prime}$ should be less than 0.1 for the batch settling effect to have a significant effect on the Stokes law. As a result the solutions with $\varepsilon^{\prime}$ over 0.1 are considered to be governed by the Stokes law alone, safely ignoring

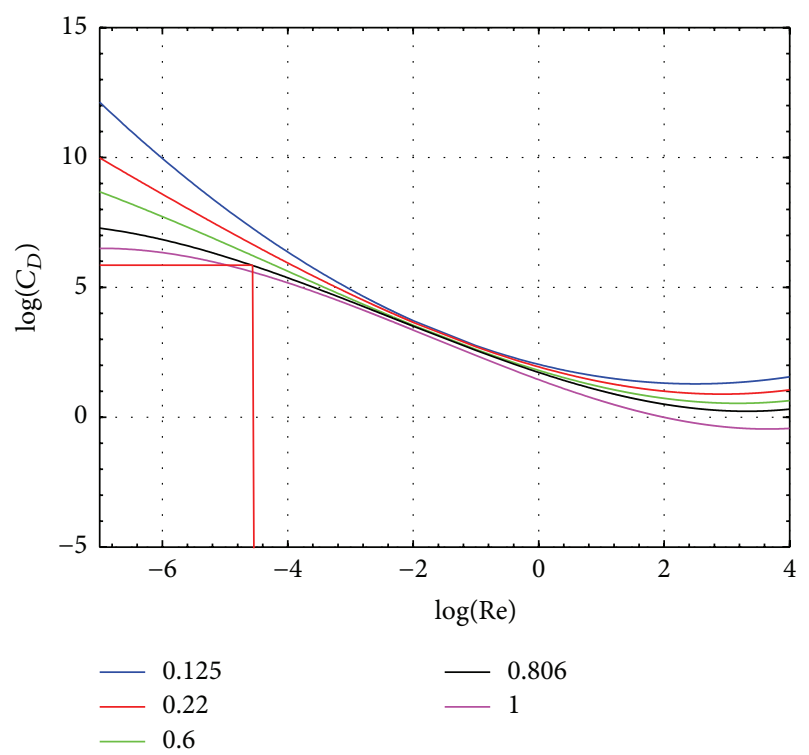

Figure 3: Drag coefficient $\left(C_{D}\right)$ for low Re and for different $\Psi$.

effect of close proximity particles. In nanofluids the particle concentrations are far smaller than this (hence $\varepsilon^{\prime}$ is much larger than 0.1). For example, in Witharana et al. [10] settling experiments, $\varepsilon^{\prime}$ was $0.723\left(\varepsilon^{\prime}=1-\emptyset_{p}\left(R_{a} / r\right)^{3-d_{f}}\right)$. Therefore, in the context of this work, the batch settling scenario is very weak. 
TABLE 2: Geometric details of different aggregate shapes. $R_{1}, R_{2}$, and $R_{3}$ are, respectively, aggregates of radii $2.5 \mu \mathrm{m}, 4 \mu \mathrm{m}$, and $5 \mu \mathrm{m}$.

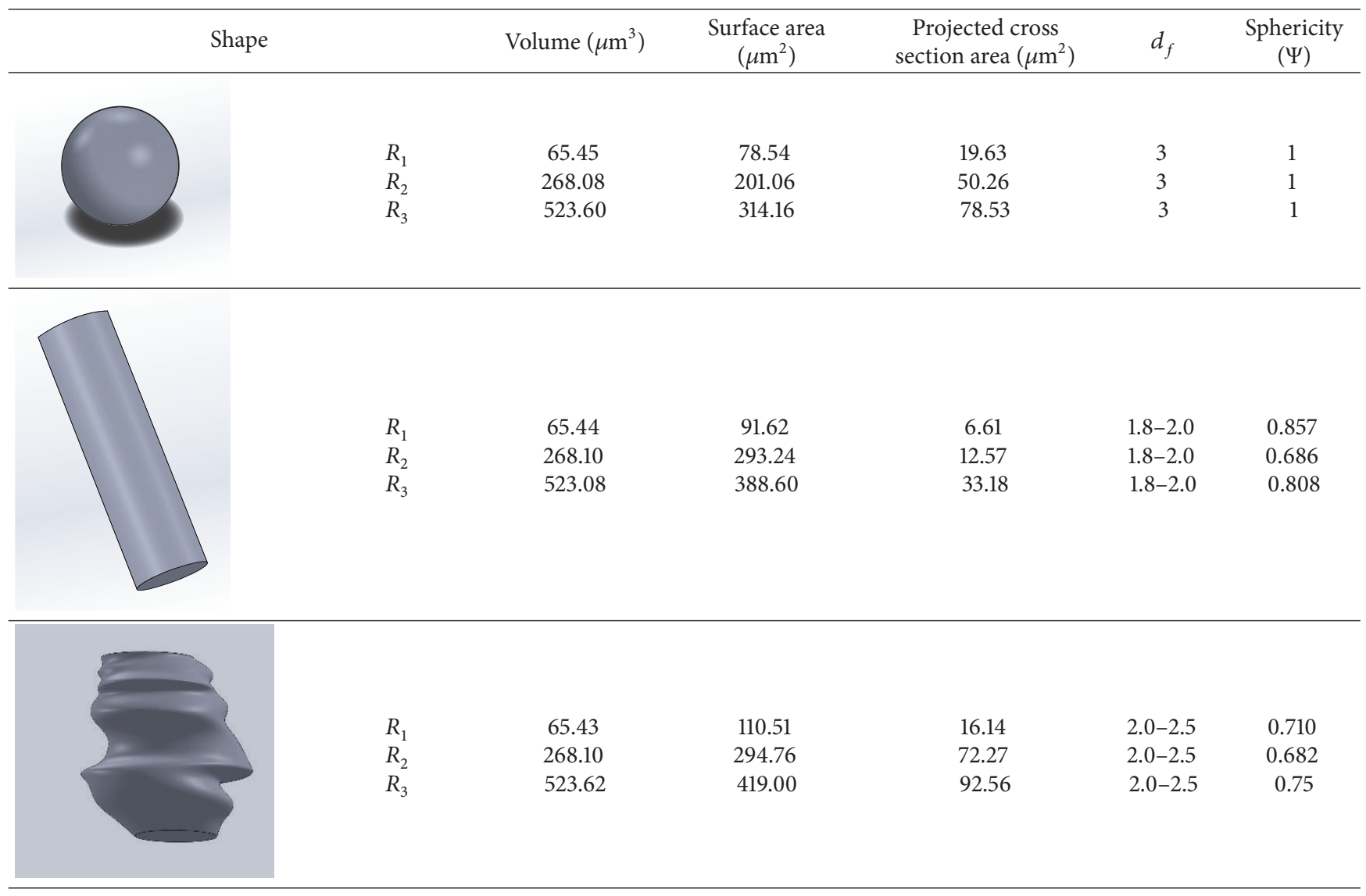

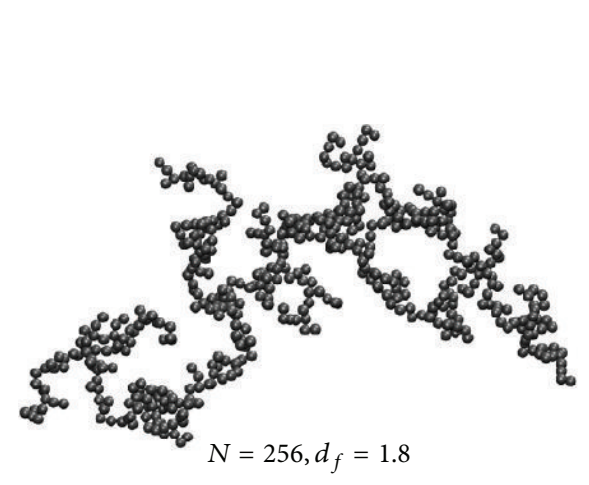

(a)

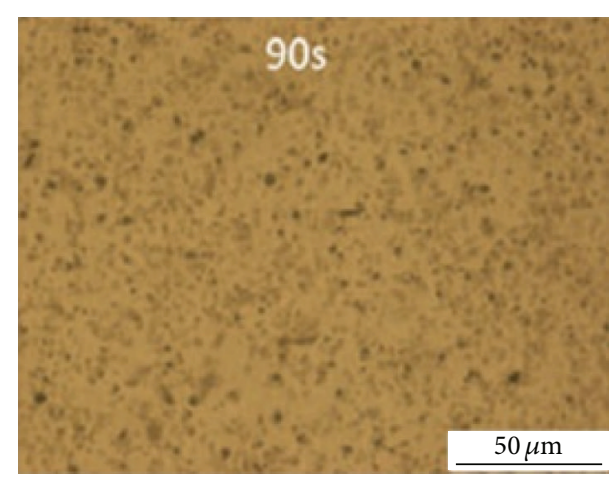

(b)

FIgURE 4: (a) Structure of an aggregate [10]. (b) Optical microscopy images of aqueous $\mathrm{Al}_{2} \mathrm{O}_{3}$ aggregates [10].

\section{Results and Discussion}

3.1. Estimation of Aggregation and Settling Times from the Proposed Model. For the validation of this model, the experimental data from Witharana et al. [10] were recruited. Their system was polydisperse spherical alumina $\left(\mathrm{Al}_{2} \mathrm{O}_{3}\right)$ nanoparticles suspended in water at near-IEP. The particle sizes were ranging within 10 100 nm verified by TEM images, with the average size of $46 \mathrm{~nm}$. From the optical microscopy images aggregates were observed to have radius in the range of $1 \mu \mathrm{m} \sim 10 \mu \mathrm{m}$, as seen from Figure 4(b). For the purpose of validating this model, the equivalent aggregate radius is taken as $2.5 \mu \mathrm{m}$, and density of $\mathrm{Al}_{2} \mathrm{O}_{3}$ nanoparticles is taken as $3970 \mathrm{~kg} / \mathrm{m}^{3}$. Based on the geometry of the aggregate shown on the microscopy images, the fractal dimension $\left(d_{f}\right)$ is estimated to be 1.8. Witharana et al's [10] nanoparticle concentration was $0.5 \mathrm{wt} \%$ which converts to equivalent volume fraction $\left(\emptyset_{p}\right)$ of $0.001 \mathrm{vol} \%$. The height of the vials where their samples were stored during the experiment was $6 \mathrm{~cm}$. 
3.1.1. Aggregation Time. At IEP, the repulsive and hydrodynamic forces become minimum and the value of $W$ tends to 1. Now, using (2) and (1), first $t_{p}$ and then $t$ are calculated for the following values: $\mu=8.92 * 10^{-4} \mathrm{~kg} / \mathrm{m} / \mathrm{s}, r_{p}=23 \mathrm{~nm}, \phi_{p}=$ $0.001, T=293 \mathrm{~K}$, and $k_{B}=1.38 \times 10^{-23} \mathrm{~J} / \mathrm{m}^{2} / \mathrm{K}^{4} / \mathrm{s}$. This yields $t_{p}=8.45 \times 10^{-3}$ s. For $R_{a}=2.5 \mu \mathrm{m}$ and $d_{f}=1.8$,

$$
t=0.65 \text { mins. }
$$

\subsubsection{Settling Velocity. Consider the following:}

(i) Density of the aggregate $\left(\rho_{\text {agg }}\right)$ is taken from Section 3 above, which was $3970 \mathrm{~kg} / \mathrm{m}^{3}$.

(ii) Density of homogenous flow $\left(\rho_{m}\right)$ is calculated from (11), which is $1003 \mathrm{~kg} / \mathrm{m}^{3}$.

(iii) Aggregation fraction $\left(\emptyset_{a}\right)$ is calculated from (12) which is 0.277 and void fraction of the liquid $\left(\varepsilon^{\prime}\right)$ is 0.723 .

(iv) Viscosity of the liquid $(\mu)$ is calculated from (13), which is $8.92 \times 10^{-4} \mathrm{~kg} \mathrm{~m}^{-1} \mathrm{~s}^{-1}$.

(v) Calculated value for $C_{D} \mathrm{Re}^{2}$ for a $5 \mu \mathrm{m}$ diameter aggregate from (9) is $6.14 \times 10^{-3}$.

(vi) Now, from Figure 1, the approximate Re is $3 \times 10^{-5}$.

(vii) Sphericity $(\Psi)$ is taken as 0.857 from Table 2 , which is in the interval of 1 and 0.806 .

(viii) From the line corresponding to 0.806 in Figure 3, the drag coefficient $\left(C_{D}\right)$ is found to be approximately $3 \times$ $10^{5}$ (indicated in Figure 3 by vertical and horizontal lines).

(ix) From (7) and assuming equivalent radius as $2.5 \mu \mathrm{m}$, terminal velocity is calculated to be

$$
4.37 \times 10^{-5} \mathrm{~m} / \mathrm{s} \text {. }
$$

(x) On the experimental study reported in [10], the actual settling velocity was stated as $6.66 \times 10^{-5} \mathrm{~m} / \mathrm{s}$. This falls within the same order of magnitude as calculated in the foregoing step (b).

3.1.3. Total Settling Time. When the suspension is at near-IEP, the nanoparticle aggregation occurs rapidly. Furthermore, as mentioned in the Section 2.2 above, it is assumed that the nanoparticles have no resultant downward motion till they get fully aggregated. This makes the total time for settling equal to the sum of aggregation time and settling time. Aggregation time was calculated in above (a) as 0.65 mins. Once aggregates were formed, assume they reached the terminal velocity in negligible time. Now the total time for settling becomes

$$
\begin{aligned}
& \text { Total time for settling } \\
& =\text { aggregation time }+ \text { settling time } \\
& =0.65 \mathrm{~min}+\frac{6 \times 10^{-2}}{4.37 \times 10^{-5}}=0.65+22.88 \\
& =23.53 \mathrm{~min} .
\end{aligned}
$$

Figure 3 in Witharana et al. [10] provides the camera images of their settling nanofluid. Close to 30 mins after preparation, their samples were completely settled. The calculated total settling time presented above is therefore in good agreement with the actual experiment reported in literature.

\section{Conclusions}

Determination of the settling rates of nano- and microparticulate systems are of both academic and industrial interest. Experimentation with a real suspension would be the most accurate method to study these complex systems. However, for industrial applications, the predictability of settling rates is of utmost importance. The equations available in literature address the sizes of submillimeter or above, leaving a gap for a predictive model that can cater to nano- and micrometer sized particles. The analytical method presented in this paper was an effort to fill this gap. To begin the procedure, one needs to know the particle concentration in the liquid. Once it is known, firstly the aggregation rates can be estimated using the modified classical correlations presented in this paper. Settling rates were then determined from a combination of equations and graphs. Total settling time thus becomes the sum of aggregation and settling times. To validate the new method, experimental data for $\mathrm{Al}_{2} \mathrm{O}_{3}$-water nanoparticulate system was recruited from literature. Their experimentally determined settling rates were $6.66 \times 10^{-5} \mathrm{~m} / \mathrm{s}$. For the same system, our model prediction was $4.37 \times 10^{-5} \mathrm{~m} / \mathrm{s}$. Thus the experimental and analytical schemes were in agreement with the same order of magnitude.

Furthermore, this analytical model is able to account for roundness deviations and fractal dimensions. However more experimental data are required to further examine the resilience of the proposed model.

\section{Conflict of Interests}

The authors declare that there is no conflict of interests regarding the publication of this paper.

\section{References}

[1] X.-F. Yang and Z.-H. Liu, "Application of functionalized nanofluid in thermosyphon," Nanoscale Research Letters, vol. 6, no. 1, article 494, 2011.

[2] H. Kim, "Enhancement of critical heat flux in nucleate boiling of nanofluids: a state-of-art review," Nanoscale Research Letters, vol. 6 , no. 1, article 415, 2011.

[3] S. Witharana, J. A. Weliwita, H. Chen, and L. Wang, "Recent advances in thermal conductivity of nanofluids," Recent Patents on Nanotechnology, vol. 7, no. 3, pp. 198-207, 2013.

[4] J. Buongiorno, D. C. Venerus, N. Prabhat et al., "A benchmark study on the thermal conductivity of nanofluids," Journal of Applied Physics, vol. 106, no. 9, Article ID 094312, 2009.

[5] S. Lotfizadeh and T. Matsoukas, "A continuum Maxwell theory for the thermal conductivity of clustered nanocolloids," Journal of Nanoparticle Research, vol. 17, no. 6, article 262, 2015.

[6] Y. T. He, J. Wan, and T. Tokunaga, "Kinetic stability of hematite nanoparticles: the effect of particle sizes," Journal of Nanoparticle Research, vol. 10, no. 2, pp. 321-332, 2008. 
[7] Y. R. Chae, Y. J. Yoon, and K. G. Ryu, "Development of modified stokes expression to model the behavior of expanded beds containing polydisperse resins for protein adsorption," Korean Journal of Chemical Engineering, vol. 21, no. 5, pp. 999-1002, 2004.

[8] H. Mann, P. Mueller, T. Hagemeier, C. Roloff, D. Thévenin, and J. Tomas, "Analytical description of the unsteady settling of spherical particles in Stokes and Newton regimes," Granular Matter, vol. 17, no. 5, pp. 629-644, 2015.

[9] S. Zhiyao, W. Tingting, X. Fumin, and L. Ruijie, "A simple formula for predicting settling velocity of sediment particles," Water Science and Engineering, vol. 1, no. 1, pp. 37-43, 2008.

[10] S. Witharana, C. Hodges, D. Xu, X. Lai, and Y. Ding, "Aggregation and settling in aqueous polydisperse alumina nanoparticle suspensions," Journal of Nanoparticle Research, vol. 14, article 851, 2012.

[11] R. Prasher, P. E. Phelan, and P. Bhattacharya, "Effect of aggregation kinetics on the thermal conductivity of nanoscale colloidal solutions (nanofluid)," Nano Letters, vol. 6, no. 7, pp. 1529-1534, 2006.

[12] H. Chen, S. Witharana, Y. Jin, C. Kim, and Y. Ding, "Predicting thermal conductivity of liquid suspensions of nanoparticles (nanofluids) based on rheology," Particuology, vol. 7, no. 2, pp. 151-157, 2009.

[13] H. Chen, S. Witharana, Y. Jin, C. Ding, and Y. Kim, "Predicting the thermal conductivity of nanofluids based on suspension viscosity," in Proceedings of the 4th International Conference on Information and Automation for Sustainability (ICIAFS '08), pp. 234-239, IEEE, Colombo, Sri Lanka, December 2008.

[14] R. L. Hamilton and O. K. Crosser, "Thermal conductivity of heterogeneous two-component systems," Industrial and Engineering Chemistry Fundamentals, vol. 1, no. 3, pp. 187-191, 1962.

[15] J. Israelachvili, "Contrasts between intermolecular, interparticle, and intersurface forces," in Intermolecular and Surface Forces, pp. 205-222, Elsevier, 3rd edition, 2011.

[16] L. H. Hanus, R. U. Hartzler, and N. J. Wagner, "Electrolyteinduced aggregation of acrylic latex. 1: dilute particle concentrations," Langmuir, vol. 17, no. 11, pp. 3136-3147, 2001.

[17] Y. Min, M. Akbulut, K. Kristiansen, Y. Golan, and J. Israelachvili, "The role of interparticle and external forces in nanoparticle assembly," Nature Materials, vol. 7, no. 7, pp. 527-538, 2008.

[18] G. Pranami, "Understanding nanoparticle aggregation," 2009, http://lib.dr.iastate.edu/cgi/viewcontent.cgi?article=1856\&context=etd.

[19] S. P. Jang and S. U. S. Choi, "Effects of various parameters on nanofluid thermal conductivity," Journal of Heat Transfer, vol. 129, no. 5, pp. 617-623, 2007.

[20] M. Rhodes, Introduction to Particle Technology, John Wiley \& Sons, Hoboken, NJ, USA, 2013.

[21] D. E. Walsh and P. D. Rao, A Study of Factors Suspected of Influencing the Settling Velocity of Fine Gold Particles, vol. 76, University of Alaska Fairbanks, Fairbanks, Alaska, 1988.

[22] J. Israelachvili, Intermolecular and Surface Forces, Elsevier, 3rd edition, 2011.

[23] T. Brownian, Brownian Motion, no. 1, Clarkson University, Potsdam, NY, USA, 2011.

[24] S. Lee, S. U.-S. Choi, S. Li, and J. A. Eastman, "Measuring thermal conductivity of fluids containing oxide nanoparticles," Journal of Heat Transfer, vol. 121, no. 2, pp. 280-288, 1999.

[25] J. C. Maxwell Garnett, "Colours in metal glasses and in metallic films," Philosophical Transactions of The Royal Society Series A:
Mathematical Physical and Engineering Sciences, vol. 203, pp. 385-420, 1904.

[26] G. Bai, W. Jiang, and L. Chen, "Effect of interfacial thermal resistance on effective thermal conductivity of $\mathrm{MoSi}_{2} / \mathrm{SiC}$ composites," Materials Transactions, vol. 47, no. 4, pp. 1247-1249, 2006.

[27] D. Walsh and P. Rao, A Study of Factors Suspected of Influencing the Settling Velocity of Fine Gold Particles, O'Neill Research Laboratory, Fairbanks, Alaska, USA, 1988.

[28] N. Visaveliya and J. M. Köhler, "Control of shape and size of polymer nanoparticles aggregates in a single-step microcontinuous flow process: a case of flower and spherical shapes," Langmuir, vol. 30, no. 41, pp. 12180-12189, 2014.

[29] L. Li, Y. Zhang, H. Ma, and M. Yang, "Molecular dynamics simulation of effect of liquid layering around the nanoparticle on the enhanced thermal conductivity of nanofluids," Journal of Nanoparticle Research, vol. 12, no. 3, pp. 811-821, 2010.

[30] B. M. Haines and A. L. Mazzucato, "A proof of Einstein's effectiveviscosity for a dilute suspension of spheres," http://arxiv.org/ abs/1104.1102.

[31] J. A. Molina-Bolívar, F. Galisteo-González, and R. HidalgoÁlvarez, "Cluster morphology of protein-coated polymer colloids," Journal of Colloid and Interface Science, vol. 208, no. 2, pp. 445-454, 1998.

[32] J. F. Richardson and W. N. Zaki, "Sedimentation and fluidisation: part I," Chemical Engineering Research and Design, vol. 75, no. 3, supplement, pp. S82-S100, 1997. 

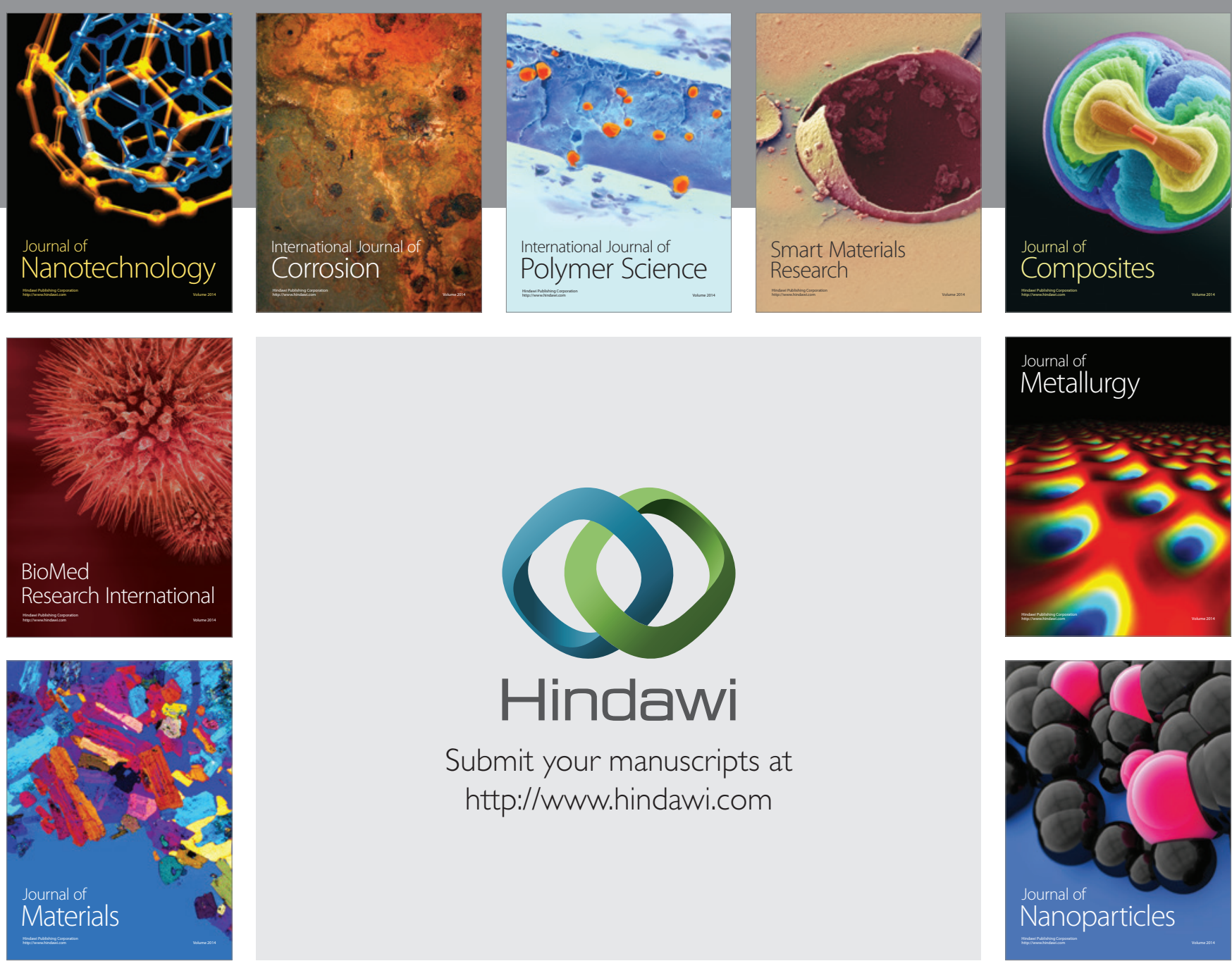

\section{Hindawi}

Submit your manuscripts at

http://www.hindawi.com

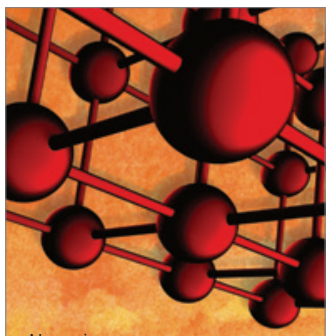

Materials Science and Engineering
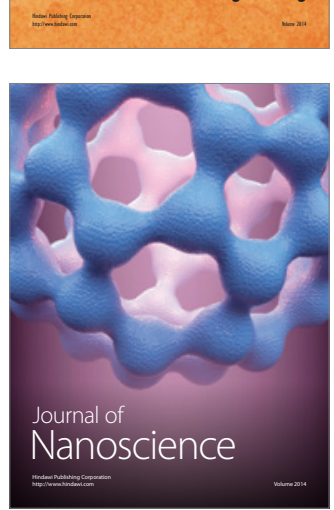
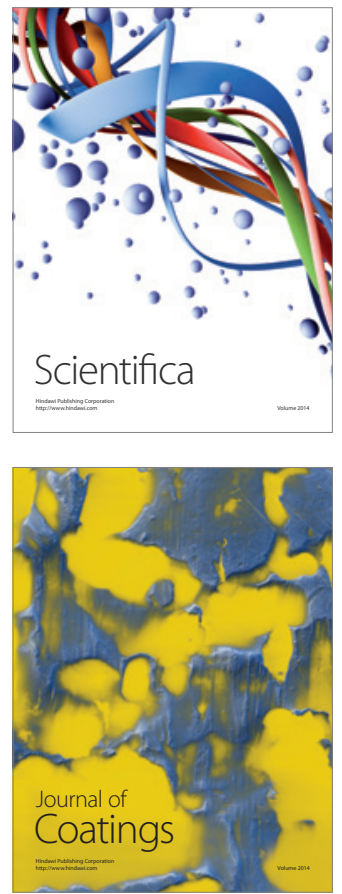
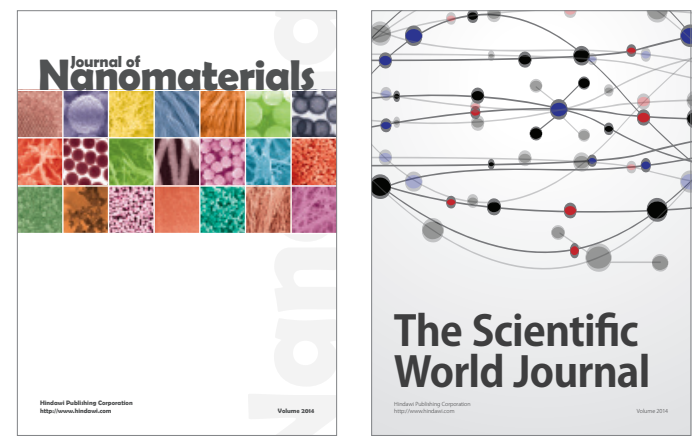

The Scientific World Journal
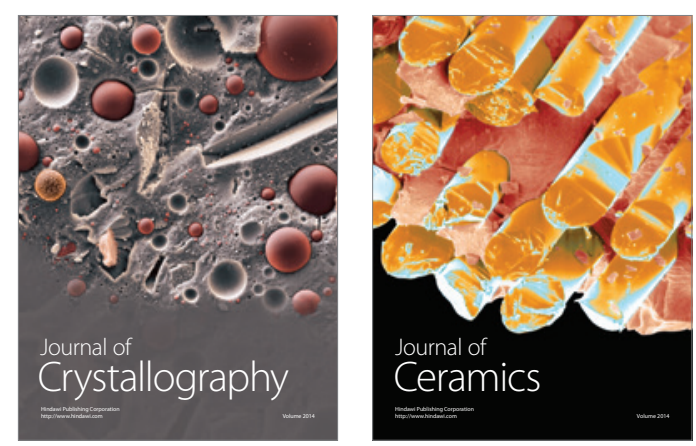
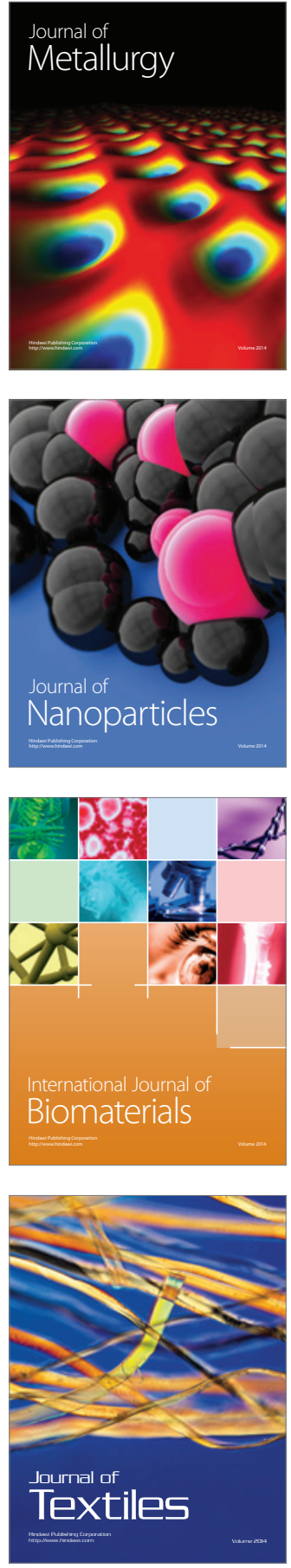